\title{
DETERMINAÇÃO DAS PROPRIEDADES MECÂNICAS DE NANOMALHAS DE GRAFENO POR SIMULAÇÃO MOLECULAR
}

\author{
A. M. CHRISTMANN e A. R. MUNIZ \\ Universidade Federal do Rio Grande do Sul, Departamento de Engenharia Química \\ E-mail para contato: amuniz@enq.ufrgs.br
}

\begin{abstract}
RESUMO -Nanomalhas de grafeno são nanoestruturas formadas a partir da inserção de poros em lâminas de grafeno, recentemente em laboratório. Aplicações em diversas áreas vêm sendo propostas, como por exemplo, em nanoeletrônica e nanofiltração. Entretanto, pouco se sabe sobre o efeito da criação destes defeitos estruturais nas propriedades mecânicas do grafeno. O objetivo deste trabalho é avaliar o impacto destas mudanças estruturais nas propriedades mecânicas do material, através de simulações de dinâmica molecular. São estimados o módulo de Young e a tensão e deformação de ruptura em testes de tração uniaxial, variando-se parâmetros estruturais do material (densidade e distribuição dos poros). Os resultados mostram que o aumento da porosidade diminui a resistência mecânica do grafeno, porém os valores estimados para as propriedades se mantém na mesma ordem de magnitude em relação ao material original, apresentando-se superior a materiais convencionais.
\end{abstract}

\section{INTRODUÇÃO}

Nas últimas décadas, tem se observado um grande interesse no desenvolvimento de materiais nanoestruturados baseados em carbono, tais como os fulerenos, os nanotubos de carbono, e mais recentemente, o grafeno. Lâminas de grafeno são materiais bidimensionais formados exclusivamente por átomos de carbono, arranjados em uma rede honeycomb, tendo um átomo de espessura. Isolado pela primeira vez em 2004 (Novoselov et al., 2004) a partir da exfoliação de grafite, o grafeno vem sendo amplamente estudado do ponto de vista fundamental e tecnológico, graças à sua excepcional combinação de propriedades físicas; um vasto campo de aplicações em potencial vem sendo sugeridas e demonstradas em diversas áreas (Novoselov et al., 2012).

No seu estado fundamental, o grafeno tem caráter semi-metálico (Novoselov et al., 2004). Para aplicações em dispositivos eletrônicos, deseja-se que o grafeno seja transformado em um semicondutor, com gaps de energia próximos ao de semicondutores convencionais. Diversas técnicas têm sido desenvolvidas em laboratório e/ou propostos teoricamente de modo a atingir este objetivo, muitos deles através da modificação estrutural do material, levando à disrupção da rede de elétrons delocalizados característico do grafeno e/ou ao confinamento espacial de elétrons. Entre estes processos destacam-se a formação de nanofitas (graphene nanoribbons) (Li et al., 2008), a inserção de defeitos estruturais de forma ordenada gerando as nanomalhas de grafeno (graphene nanomeshes) (Bai et al., 2010; Liang et al., 2010) e a funcionalização química (Elias et al., 2009). 


\section{9 a 22 de outubro de 2014 \\ Florianópolis/SC}

A manipulação e controle de propriedades físicas através da inserção de defeitos estruturais é atrativa devido à existência de tecnologias de microfabricação tradicionais em microeletrônica que podem ser usadas para tanto. Estudos teórico-computacionais mostram que com a criação de defeitos arranjados de forma regular, levando a criação de superestruturas periódicas, conforme ilustrado na Figura 1, é possível controlar com precisão das propriedades eletrônicas e magnéticas do material (Pedersen et al., 2008). Dependendo da densidade e distribuição espacial dos defeitos, o material resultante pode ser um metal, semi-metal, ou um semicondutor, com grande variação no gap eletrônico obtido. Experimentos corroboram estes resultados (Bai et al., 2010; Liang et al., 2010)

Além do controle de propriedades eletrônicas, a criação de nanomalhas de grafeno possui outra aplicação em potencial, que é a de atuar como uma peneira/membrana molecular. Uma série de estudos mostram a viabilidade e a eficiência do uso de nanomalhas de grafeno na purificação de gases e separação de isótopos (Jiang e Fan, 2014). O controle das dimensões dos poros na síntese da nanomalha permite com que diferentes graus de separação possam ser atingidos.

Apesar do grande interesse nestas estruturas e no desenvolvimento de aplicações, pouco se sabe sobre o efeito da presença destes defeitos nas propriedades mecânicas do material, quando comparado ao grafeno, que por sua vez, apresenta excelente resistência mecânica. Do ponto de vista qualitativo, espera-se que haja uma perda na resistência mecânica, sendo maior esta perda quanto maior a presença de defeitos. Estudos recentes abordaram este aspecto, e mostram que há uma diminuição no módulo de elasticidade do material (Sahin e Ciraci, 2011; Mostério e Fonseca, 2013; Carpenter et al., 2014). Estes estudos foram limitados a sistemas de pequena dimensão, devido a limitações no custo computacional inerentes ao método empregado, e exploraram essencialmente o regime de deformação linear; o fenômeno de fratura (altas deformações) não foi investigado nestes estudos anteriores.

Neste contexto, o presente trabalho tem o objetivo de analisar de forma sistemática a influência da porosidade nas propriedades mecânicas de nanomalhas de grafeno, determinadas em testes de tração uniaxial usando simulações de dinâmica molecular clássica. Será investigado como as propriedades mecânicas estimadas - módulo de elasticidade, tensão de ruptura e deformação de ruptura - dependem da porosidade do material e de parâmetros geométricos da estrutura, tais como o tamanho dos poros e da periodicidade da superestrutura. Mostra-se que realmente há um decréscimo nas propriedades mecânicas do material, entretanto, os valores se mantém ainda elevados quando comparados a materiais convencionais em uma ampla faixa de porosidade. Observou-se uma mudança no comportamento mecânico em determinado intervalo do parâmetro investigado. O trabalho está estruturado da seguinte forma: na seção 2, a metodologia computacional é brevemente descrita; resultados são apresentados e discutidos na seção 3, e conclusões são mostradas na seção 4.

\section{METODOLOGIA COMPUTACIONAL}

Para condução das simulações, estruturas atômicas das nanomalhas com diferentes porosidades e periodicidades foram obtidas a partir da remoção de átomos de uma lâmina de grafeno previamente relaxada, de modo a criar uma superestrutura periódica. As superestruturas geradas apresentam simetria hexagonal, a mesma observada em nanomalhas obtidas experimentalmente (Bai et al,, 2010). Um exemplo de estrutura gerada é mostrado na Figura 1, destacando as três dimensões características 




19 a 22 de outubro de 2014
Florianópolis/SC

de cada nanomalha: $D$ é o diâmetro médio dos poros, $d$ é a distância entre dois poros consecutivos ("pescoço") e $P=D+d$ é a periodicidade da superestrutura. Para geração das nanomalhas, empregaram-se neste estudo duas supercélulas periódicas de tamanhos distintos, que serão tratadas por SC_1, contendo 9248 átomos (na ausência de defeitos) e de dimensão 16,7 nm × 14,5 nm, e por SC_2, contendo 36992 átomos (na ausência de defeitos) e de dimensão 33,5 nm $\times$ 29,0 nm. Com estas estruturas foi possível investigar nanomalhas com diâmetros na faixa de $5 \AA$ - $76 \AA$ e periodicidade na faixa de $38,4 \AA-83 \AA$.

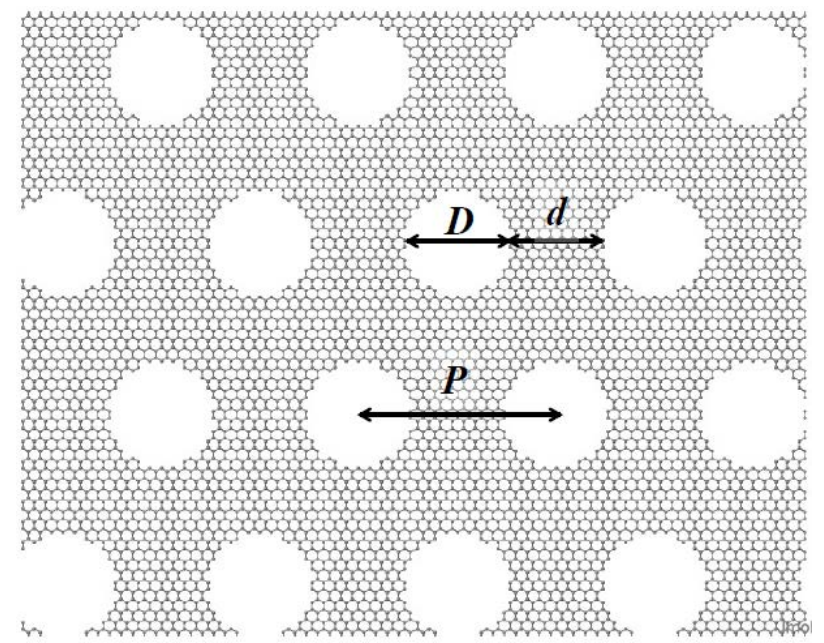

Figura 1 - Exemplo de uma configuração atômica de uma nanomalha gerada a partir de uma lâmina de grafeno. As dimensões características da nanoestrutura $(D=$ diâmetro dos poros, $d=$ pescoço e $P=$ periodicidade) são indicadas na figura.

As propriedades mecânicas de nanomalhas de grafeno em testes de tração uniaxial foram estimadas usando simulações de dinâmica molecular (MD) clássica, empregando o pacote LAMMPS (Plimpton, 1995). Simulações de dinâmica molecular consistem na resolução das equações do movimento para $N$ corpos clássicos ( $N$ átomos da estrutura) que interagem de acordo com uma função de potencial interatômico. O potencial utilizado para descrição das interações interatômicas foi o AIREBO (Stuart et al., 2000), amplamente empregado em simulações envolvendo alótropos de carbono em fase sólida. O termostato/barostato de Berendsen (Berendsen et al., 1984) foi utilizado para controle de pressão e temperatura. Condições de contorno periódicas foram aplicadas nas três direções (de forma a representar um sistema infinito). Nos testes de tração uniaxial, as simulações foram conduzidas sob taxa de deformação e temperatura constantes $\left(0,0001 \mathrm{ps}^{-1}\right.$ e $\left.300 \mathrm{~K}\right)$, mantendose pressão hidrostática na direção oposta à deformação, sendo que as estruturas são previamente relaxadas na temperatura de trabalho por um período de tempo adequado. Durante o processo de deformação, calcula-se em determinados intervalos de tempo a tensão acumulada na estrutura, obtendo-se assim curvas de tensão-deformação, como ilustradas na Figura 2. Três propriedades mecânicas são extraídas a partir destas curvas: no ponto de ruptura (verificada pela queda brusca da tensão) obtém-se a tensão e deformação de ruptura, e na região referente a baixas deformações (regime linear), a inclinação da curva corresponde ao módulo de Young. Devido ao caráter anisotrópico das nanoestruturas, a deformação foi aplicada em duas direções independentes ( $x$ e $y$ ). 


\section{RESULTADOS E DISCUSSÃO}

Foram geradas diversas nanomalhas usando o procedimento descrito na Seção 2, variando-se o diâmetro dos poros e a periodicidade da superestrutura. De forma a generalizar os resultados obtidos e possibilitar a extrapolação de resultados para nanomalhas de diferentes dimensões características, foi definida a variável adimensional $\phi_{\mathrm{D}}=D / P=D /(d+D)$, que consiste na razão entre o diâmetro do poro e a periodicidade da superestrutura, tal que $0<\phi_{\mathrm{D}}<1$. Esta variável está diretamente correlacionada com a porosidade do material.

A Figura 2 mostra algumas curvas de tensão versus deformação que foram obtidas nas simulações de dinâmica molecular. Os resultados apresentados nesta figura demonstram que as nanomalhas comportam-se como um material frágil sob tensão uniaxial, com um ponto de ruptura bem definido, analogamente ao grafeno e outras nanoestruturas de carbono (Pei et al., 2010). Observa-se que há um enfraquecimento do material com a inserção de poros, comparado ao grafeno. Verifica-se também que a tensão e deformação de ruptura dependem das dimensões características da malha; primeiramente com um aumento do tamanho relativo dos poros, as propriedades decrescem. Porém, com o aumento deste parâmetro a partir de um determinado valor, verifica-se que mesmo que o módulo de Young e a tensão de ruptura decresçam, a deformação requerida para a ruptura começa a aumentar, ou seja, o material suporta menos carga, mas deforma mais antes de romper. Uma mudança na concavidade da curva também é observada.

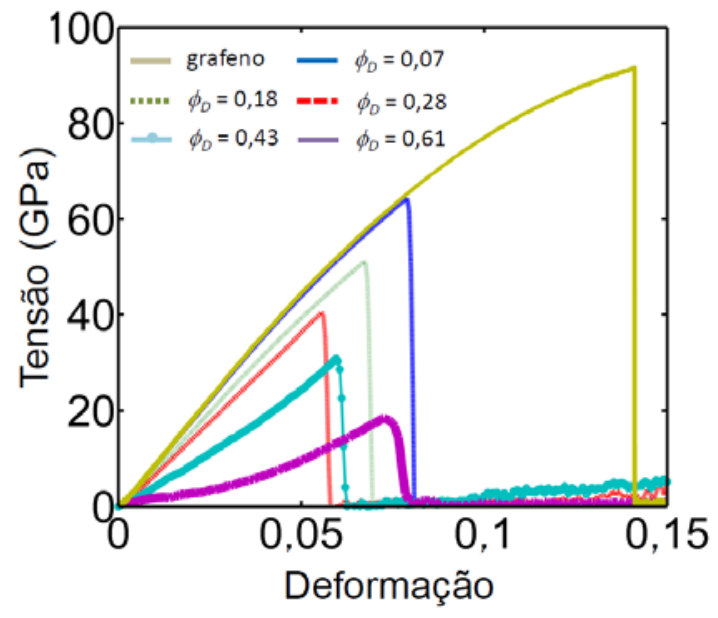

Figura 2 - Curvas de tensão versus deformação obtidas em simulações de dinâmica molecular referentes a testes de tração uniaxial para uma lâmina de grafeno e diferentes nanomalhas, com deformação aplicada na direção $y$.

A Figura 3 mostra configurações atômicas em diferentes instantes da simulação da deformação uniaxial de uma nanomalha com $\phi_{D}=0,37$, ao longo da direção $x$. São apresentadas configurações em diferentes estados de deformação, antes e após a fratura. Os átomos estão coloridos de acordo com a tensão atômica local, o que permite analisar a distribuição e tensões na estrutura e identificar os pontos onde a fratura tem maior probabilidade de se iniciar. Verifica-se a concentração de tensões em 

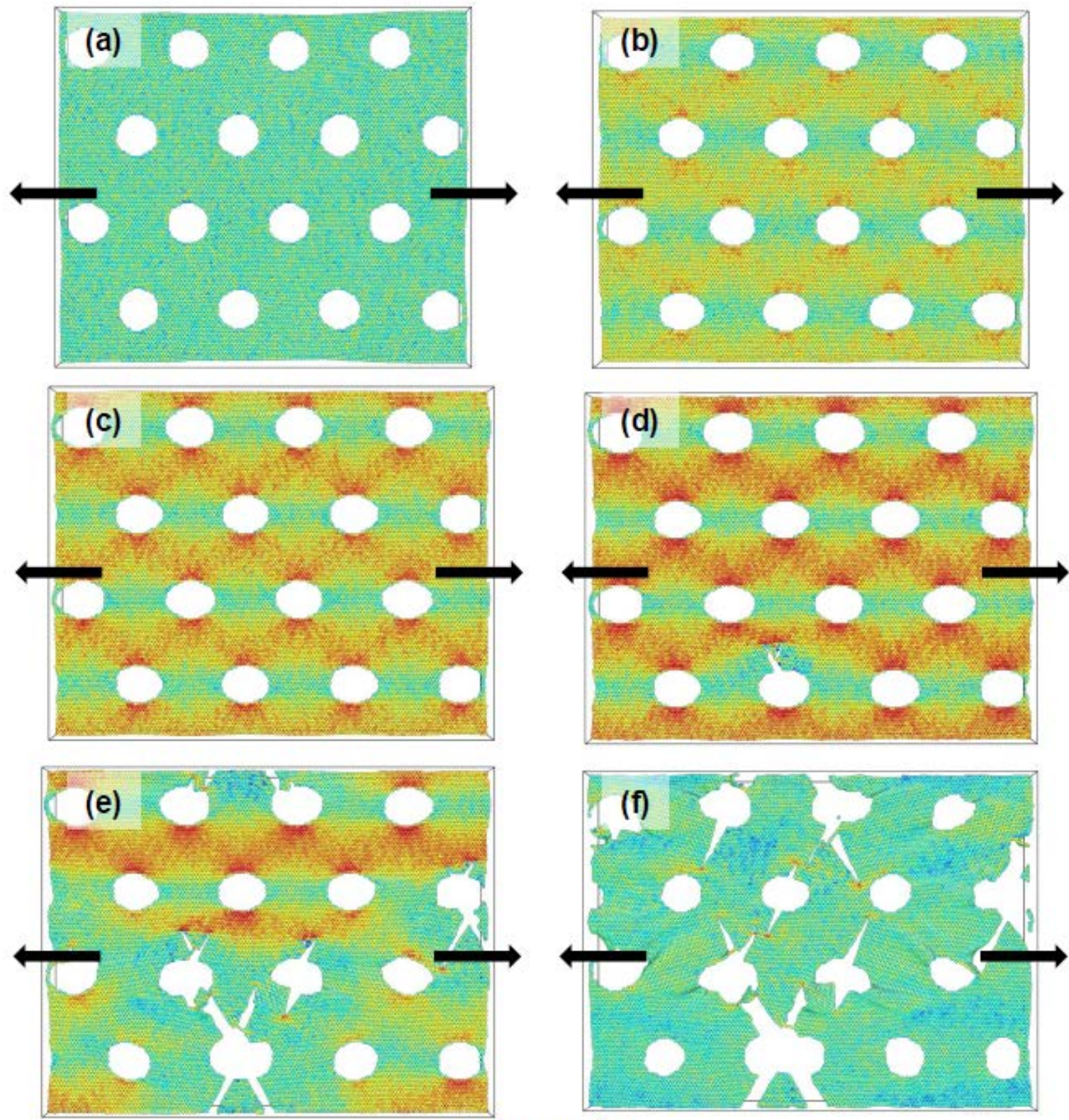

$\tau_{x x} \Omega\left(\operatorname{bar} \AA^{3}\right)$

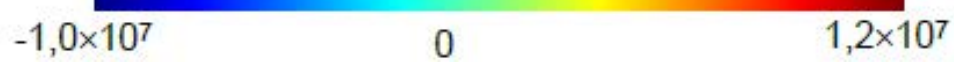

Figura 3 -Configurações instantâneas de uma simulação MD do teste de tração uniaxial aplicada à direção $x$ para uma nanomalha com $\phi_{D}=0,37$, de 33,5 $\mathrm{nm} \times 29,0 \mathrm{~nm}$. Os átomos estão coloridos de acordo com a componente $\tau_{x x}$ do tensor das tensões multiplicado pelo volume atômico $\Omega$, seguindo a legenda dada na parte inferior da figura. São mostradas configurações atômicas referentes à estrutura

(a) inicial (na temperatura de teste), (b) levemente tensionada, (c) altamente tensionada, (d) no instante onde o primeiro crack é formado, e (e-f) em instantes onde os cracks formados aumentam e se propagam ao longo da estrutura. 


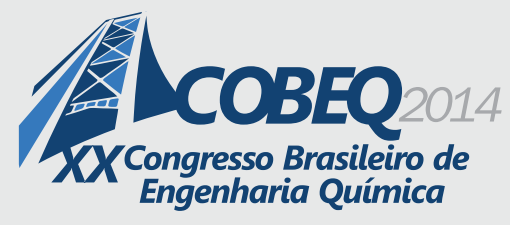

19 a 22 de outubro de 2014
Florianópolis/SC

linhas diagonais conectando os poros, especialmente junto às arestas superior e inferior dos poros. Portanto, a inserção de poros leva a formação de interfaces que concentram tensões; nestes pontos de concentração de tensões, observa-se que com o aumento da tensão normal aplicada, forma-se um crack inicial, que se propaga preferencialmente ao longo das linhas de concentração de tensões. Comportamento análogo foi observado para outras estruturas.

A dependência das propriedades mecânicas no parâmetro $\phi_{D}$, pode ser melhor observada na Figura 4. Dados obtidos para nanomalhas geradas a partir das duas distintas supercélulas (SC_1 e SC_2), e para deformações aplicadas nas duas direções, são mostradas no mesmo gráfico. É evidente a dependência entre as propriedades estimadas e o parâmetro adimensional $\phi_{D}$, independentemente das dimensões absolutas dos poros e periodicidade da superestrutura. Desta forma, os resultados apresentados podem ser estendidos a nanomalhas de diferentes dimensões, como por exemplo, às obtidas experimentalmente reportadas na literatura (Bai et al., 2010; Liang et al., 2010). Verifica-se também que os resultados são independentes do tamanho do sistema, atestando a convergência dos resultados neste parâmetro. A anisotropia da estrutura tem pouco efeito comparado ao parâmetro $\phi_{D}$.

Para o módulo de Young (Figura 4(a)), verifica-se um decréscimo linear com aumento da porosidade refletido pelo parâmetro $\phi_{D}$, até um valor de $\sim 0,6$; a partir deste valor, o módulo decresce suavemente até atingir o valor limite, em torno de $10 \mathrm{GPa}$. Na Figura 4(b), verifica-se que a tensão de ruptura decresce com $\phi_{D}$, até um valor crítico de $\sim 0,6$ (mesmo ponto onde há a mudança no comportamento do módulo de elasticidade), sendo que a partir deste ponto, se mantém praticamente invariável com aumento da porosidade. Na Figura 4(c) observa-se que a deformação de ruptura decresce bruscamente com o aumento da porosidade, se mantendo constante por uma determinada faixa do parâmetro adimensional $\phi_{D}$, $(0,10-0,60)$, passando a aumentar com $\phi_{D}$ após este valor crítico.

Analisando as trajetórias detalhadas das simulações, verificou-se que a partir deste valor crítico de $\phi_{D}=0,6$, há uma mudança significativa na morfologia da superfície, tal que a estrutura se contrai na direção perpendicular à deformação, fazendo com que haja uma redistribuição nas tensões ao longo do sistema, aliviando a tensão nos pontos críticos (conforme mostrado na Figura 3), e aumentando assim, a deformação necessária para ruptura. Estudos estão sendo conduzidos para melhor compreensão deste fenômeno, e serão reportados em um trabalho futuro.

\section{CONCLUSÕES}

Este trabalho apresenta os resultados de uma análise computacional das propriedades mecânicas de nanomalhas de grafeno. Usando simulações de dinâmica molecular, verifica-se que as propriedades são reduzidas em relação ao material original (grafeno), porém se mantém altas em uma grande faixa de porosidade, superior às correspondentes de materiais convencionais. Os resultados são expressos em termos de um parâmetro adimensional para descrição da porosidade do material, e verifica-se uma dependência clara das propriedades em relação a estes parâmetros, sendo que os resultados podem ser extrapolados para nanomalhas de dimensões distintas das estudadas. Observouse o mecanismo de fratura típico desta estrutura, e verificou-se uma mudança no comportamento mecânico do material em estruturas com alta porosidade. 
(a)

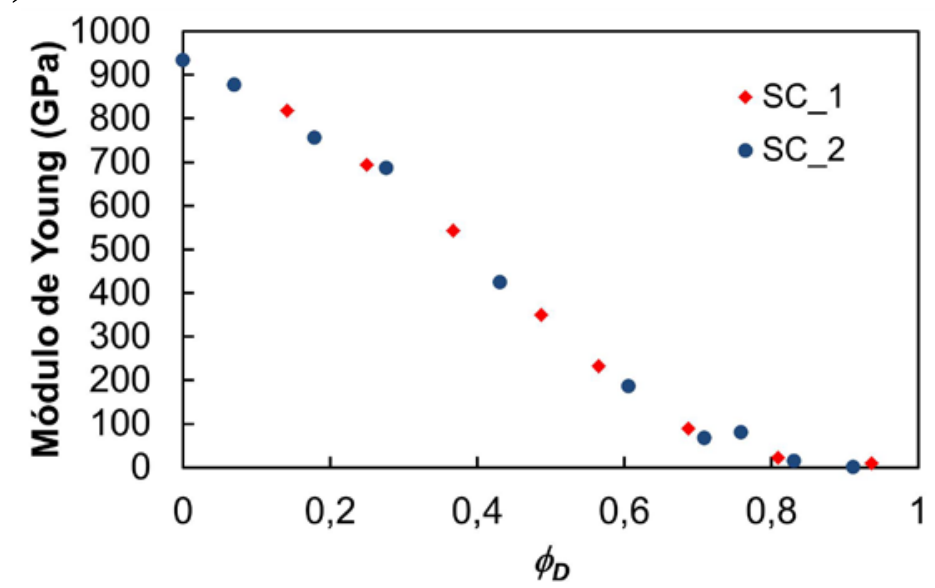

(b)

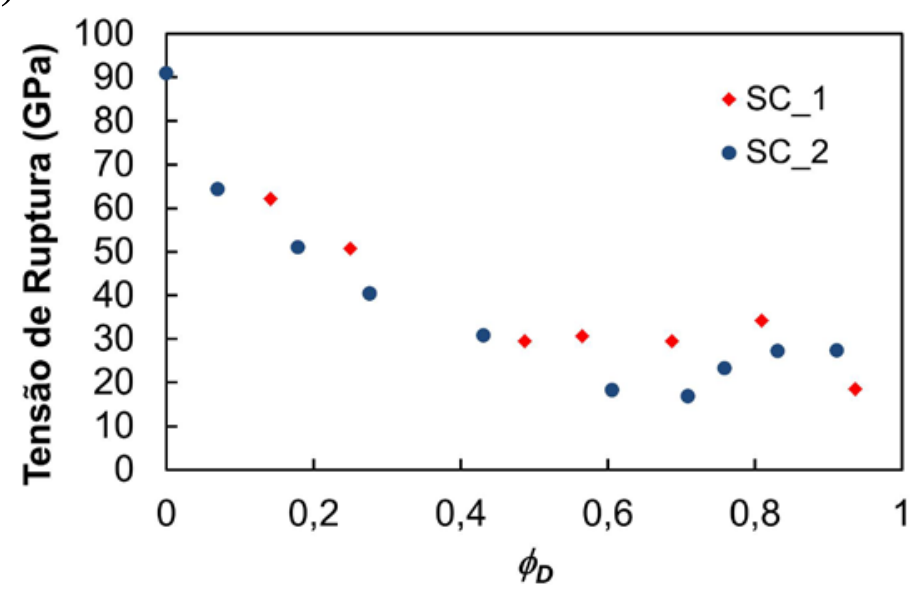

(c)

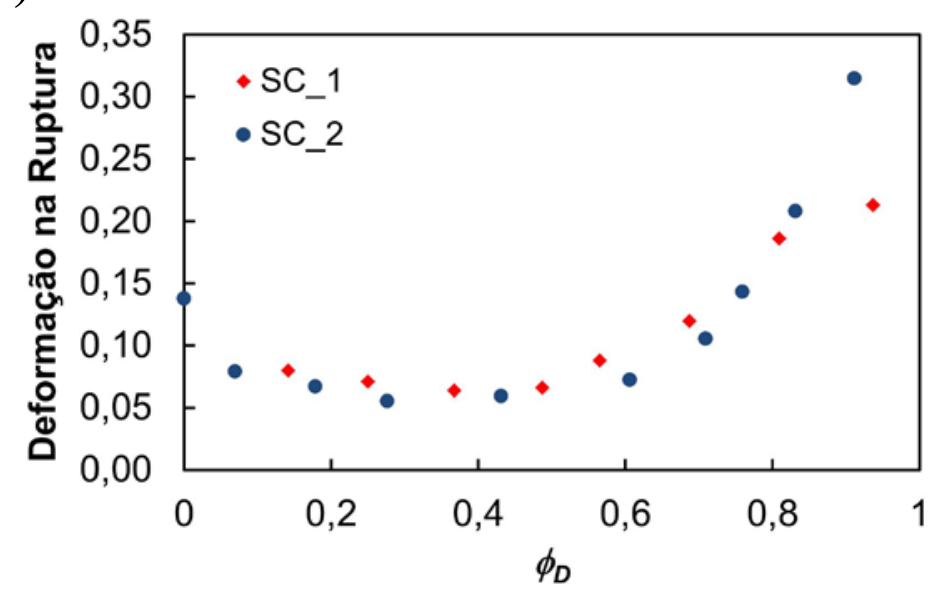

Figura 4 - Propriedades mecânicas estimadas como função do parâmetro $\phi_{\mathrm{D}}$ : (a) módulo de Young, (b) tensão de ruptura e (c) deformação de ruptura. 


\section{REFERÊNCIAS}

BAI, J.; ZHONG, X.; JIANG, S.; HUANG, Y.; DUAN, X. Graphene nanomesh. Nature Nanotech, v. 5, p. 190-194, 2010.

BERENDSEN, H.J.C.; POSTMA, J.P.M.; VAN GUNSTEREN, W.F.; DINOLA, A.; HAAK, J.R. Molecular dynamics with coupling to an external bath. J. Chem. Phys., v. 81, p. 84-90, 1984.

CARPENTER, C.; CHRISTMANN, A.M.; HU, L.; FAMPIOU, I.; MUNIZ, A.R.; RAMASUBRAMANIAM, A.; MAROUDAS, D. Elastic properties of graphene nanomeshes. Appl. Phys. Lett., v. 104, 141911, 2014.

ELIAS, D.C.; NAIR, R.R.; MOHIUDDIN, T.M.G.; MOROZOV, S.V.; BLAKE, P.; HALSALL, M.P. Control of graphene's properties by reversible hydrogenation: evidence for graphane. Science, v. 323, p. 610-613, 2009.

JIANG, L.; FAN, Z. Design of advanced porous graphene materials: from graphene nanomesh to 3D architectures. Nanoscale, v. 2, p. 1922-1945, 2014.

LI, X.; WANG, X.; ZHANG, L.; LEE, S.; DAI, H. Chemically Derived, Ultrasmooth Graphene Nanoribbon Semiconductors. Science, v. 319, p. 1229-1232, 2008.

LIANG, X; JUNG, Y.S.; WU, S.; ISMACH, A.; OLYNICK, D.L.; CABRINI, S.; BOKOR, J. Formation of Bandgap and Subbands in Graphene Nanomeshes with Sub-10 nm Ribbon Width Fabricated via Nanoimprint Lithography. Nano Lett., v.10, p. 2454-2460, 2010.

MOSTÉRIO, N.C.B; FONSECA, A.F. Mechanical and Thermal Properties of Graphene Nanomeshes. MRS Proceedings, v. 1505, 2013.

NOVOSELOV, K.S.; FALKO, V.I.; COLOMBO, L.; GELLERT, P.R.; SCHWAB, M.G.; KIM, K. A roadmap for graphene. Nature, v. 490, p. 192-200, 2012.

NOVOSELOV, K.S.; GEIM, A.K.; MOROZOV, S.V.; JIANG, D.; ZHANG, Y.; DUBONOS, S.V.; GRIGORIEVA, I.V.; FIRSOV, A.A. Electric Field Effect in Atomically Thin Carbon Films. Science, v. 306, p. 666-669, 2004.

PEDERSEN, T.G.; FLINDT, C.; PEDERSEN, J.; MORTENSEN, N.A.; JAUHO, A-P. PEDERSEN K. Graphene Antidot Lattices: Designed Defects and Spin Qubits. Phys. Rev. Lett., v. 100, 136804-14, 2008.

PEI, Q.X.; ZHANG, Y.W.; SHENOY, V.B. A molecular dynamics study of the mechanical properties of hydrogen functionalized graphene. Carbon, v. 48, p. 898-904, 2010.

PLIMPTON, S.J. Fast parallel algorithms for short-range molecular dynamics. J. Comp. Phys., v. 117, p. 1-19, 1995.

ŞAHIN, H.; CIRACI, S. Structural, mechanical, and electronic properties of defect-patterned graphene nanomeshes from first principles. Phys. Rev. B, v. 84, 035452, 2011.

STUART, S.J.; TUTEIN, A.B.; HARRISON, J.A. A reactive potential for hydrocarbons with intermolecular interactions. J. Chem. Phys., v. 112, p. 6472-86, 2000. 